\title{
Lipocalin-2 abrogates epithelial cell cycle arrest by PPARy inhibition
}

\author{
Michaela Jung ${ }^{1} \cdot$ Bernhard Brüne $^{1} \cdot$ Andreas von Knethen $^{1} \cdot$ Roser Guiteras $^{2} \cdot$ Josep Maria Cruzado ${ }^{2}$. \\ Georgina Hotter ${ }^{3,4}$. Anna Sola ${ }^{2,4}$
}

Received: 14 June 2017 / Revised: 25 May 2018 / Accepted: 5 June 2018 / Published online: 7 August 2018

(c) United States \& Canadian Academy of Pathology 2018

\begin{abstract}
Macrophage-epithelial cross-talk regulates cell cycle progression and represents an important factor in rescuing epithelial cells from cell cycle arrest in order to maintain a healthy epithelial phenotype. However, the underlying mechanisms are still not well defined. We provide evidence that macrophage-secreted lipocalin-2 (Lcn-2) plays a key role during this process. In a co-culture setup using cell cycle arrested NRK52e renal epithelial cells and primary bone marrow-derived macrophages, Lcn-2 restores proliferation through inhibition of peroxisome proliferator-activated receptor (PPAR)- $\gamma$. Lcn-2 overexpression in macrophages overcomes epithelial cell cycle arrest and enhances epithelial markers via megalin and the downstream activation of PI3K/Akt signalling pathway, whereas a knockdown of Lcn-2 in macrophages prevented this effect. Our results show that macrophage-secreting Lcn-2 is crucial in rescuing epithelial cells from cell cycle arrest and in promoting epithelial proliferation.
\end{abstract}

\section{Introduction}

Epithelial damage is seen in a number of pathologies, including inflammatory diseases, ischaemic events and/or following mechanical injury. Efficient epithelial repair is critical in re-establishing homoeostasis and is orchestrated by a network of mediators generated by the epithelium and inflammatory cells, resulting in resolution of inflammation with accompanying removal of damaged cells, subsequent proliferation, and remodelling [1].

These authors contributed equally: Georgina Hotter, Anna Sola.

Electronic supplementary material The online version of this article (https://doi.org/10.1038/s41374-018-0098-4) contains supplementary material, which is available to authorized users.

Anna Sola

asola@idibell.cat

1 Institute of Biochemistry I, Goethe-University Frankfurt, TheodorStern-Kai 7, 60590 Frankfurt, Germany

2 Department of Experimental Nephrology, IDIBELL, L'Hospitalet del Llobregat, Barcelona, Spain

3 Department of Ischemia and Inflammation, IIBB-CSIC-IDIBAPS, Rosselló, 161, 7th floor, 08036 Barcelona, Spain

4 CIBER-BBN, Networking Centre on Bioengineering, Biomaterials and Nanomedicine (CIBER-BBN), Barcelona, Spain
Epithelial repair has been extensively studied in a variety of organs, among others the skin, intestinal mucosa, or the kidney. Renal macrophages promote repair and regeneration after injury primarily through paracrine effectors or a cell-cell cross-talk [2]. Macrophage-derived Wnt7b accelerates epithelial tubule recovery via Wnt- $\beta$-catenin signalling [3] and stimulates repair of renal epithelial cells after ischaemic injury [4]. In lung, macrophages induce alveolar repair via the secretion of epithelial GM-CSF [5], while in pancreas, macrophage-epithelial cell cross-talk regulates cell cycle progression [6]. Despite these recent advances, the mechanisms involved in rescuing epithelial cell cycle arrest during the injury phase of organ damage and the subsequent induction of proliferation are not well understood.

We previously described macrophage-derived lipocalin-2 (Lcn-2) as a pro-proliferative mediator [7]. Lcn-2 is a 25 $\mathrm{kDa}$ protein of the lipocalin superfamily [8] that is crucial in defining repair and regeneration outcome, depending on the inflammatory microenvironment. Blocking Len-2 production in macrophages reduces the protective effects of IL-10cell therapy [9]. Recently, we observed that Lcn-2 overexpression in macrophages prevents renal damage in ischaemia/reperfusion injury (IRI)-sensitive rats, whereas macrophage Lcn-2-deficiency in IRI-resistant rats promotes renal cell apoptosis and delays renal regeneration [10]. Other authors demonstrated that Lcn-2 significantly promotes human pulmonary artery smooth muscle cell 
proliferation by activating the PI3K/Akt-pathway [11]. Furthermore, Lcn-2 is a modulator of peroxisome proliferator-activated receptor (PPAR)- $\gamma$ activation and has a proven role in lipid homoeostasis and energy expenditure $[12,13]$. PPAR- $\gamma$ activation downregulates cell proliferation by inhibiting Akt phosphorylation and its downstream targets [14]. Taking these data into consideration, we asked whether Lcn-2 activates epithelial proliferation through inhibition of PPAR- $\gamma$ following epithelial cell cycle arrest. Our results indicate that Lcn-2 plays a crucial role in epithelial proliferation by attenuating activation of PPAR $\gamma$ and in the induction of epithelial marker expression via activating megalin and the downstream activation of PI3K/Akt signalling pathway.

\section{Materials and methods}

\section{Materials}

The PPAR $\gamma$ agonist rosiglitazone and the PPAR $\gamma$ antagonist GW9662 were a kind gift from Prof. Dr. von Knethen (Institute for Biochemistry I, Goethe-University Frankfurt, Germany). Conditioned medium from macrophages was supplemented and incubated with the PPAR $\gamma$ agonist (1 $\mu \mathrm{M})$ or the antagonist $(100 \mathrm{nM})$ for $30 \mathrm{~min}$ prior to stimulating NRK-52e cells with conditioned medium for $24 \mathrm{~h}$. Neutralizing anti-LCN-2 and an isotype control anti-IgG were purchased from R\&D (Madrid, Spain). Cell culture supplements and FCS were ordered from Gibco (Darmstadt, Germany). Primers were bought from Invitrogen (Barcelona, Spain). The siRNA against Lcn-2 and scrambled, nontargeting control siRNA were from Invitrogen. All chemicals were of the highest grade of purity and commercially available. LY294002 was ordered from Alexis Biochemical (Lörrach, Germany). A loss of cell viability was excluded for cell treatments based on trypan blue staining (Biochrom AG, Berlin, Germany).

\section{Renal epithelial cell culture}

Rat renal epithelial cells NRK-52e (European Collection of Cell Culture, Salisbury, UK) were cultured in Dulbecco's modified Eagle Medium (DMEM) with high glucose, $15 \mathrm{mM}$ HEPES and L-glutamine, supplemented with $100 \mathrm{U} / \mathrm{ml}$ penicillin, $100 \mu \mathrm{g} / \mathrm{ml}$ streptomycin, and $10 \%$ FCS. Cells were kept in a humidified atmosphere of $5 \% \mathrm{CO}_{2}$ in air at $37^{\circ} \mathrm{C}$ and were passaged every second day using $1 \mathrm{mM}$ EDTA/0.025\% trypsin (Gibco).

Cell cycle arrest was performed as described previously [15]. Briefly, $50 \mu \mathrm{M}$ colchicine (Sigma, Madrid, Spain) was added to NRK-52e cells for $24 \mathrm{~h}$ to decrease proliferation without compromising viability. Colchicine was removed, fresh medium was added and cell viability was checked. Individual stimuli were applied for another $24 \mathrm{~h}$.

\section{Primary macrophage generation}

Rat bone marrow-derived macrophages (BMDM) were isolated from Sprague Dawley rats (Charles River, Barcelona, Spain) by aspiration of the femur, re-suspended in DMEM, supplemented with $10 \%$ FCS, $10 \mathrm{ng} / \mathrm{ml}$ GM-CSF (Peprotech, Hamburg, Germany), $100 \mathrm{U} / \mathrm{ml}$ penicillin, and $100 \mu \mathrm{g} / \mathrm{ml}$ streptomycin, and kept in non-adherent culture flasks for 7 days. Macrophages were subsequently isolated by adherence and purity was determined by ED1 and CD11b expression $[16,9,10]$.

\section{Preparation of macrophage conditioned media}

For the generation of conditioned media, cells were serumstarved for $24 \mathrm{~h}$. Primary rat macrophages were then transfected either with siRNA to knockdown Lcn-2 (siMACS), a scrambled control siRNA (scMACS), with adenoviral vectors to overexpress Lcn-2 (Lcn-2-MACS) or a control vector expressing $\beta$-galactosidase ( $\beta$-gal-MACS). After removing the supernatant, macrophages were washed twice with PBS, and incubated for another $24 \mathrm{~h}$ with DMEM without serum. The resultant supernatant was termed conditioned medium (CM) and subsequently used in further experiments as indicated. CM was collected by centrifugation $(13,000 \times \mathrm{g}, 10 \mathrm{~min})$ and filtered through $0.2 \mu \mathrm{m}$ pore filters (Millipore, Madrid, Spain) to remove large particles.

\section{Renal epithelial cell/macrophage co-culture}

Co-culture of NRK-52e cells and primary macrophages at a ratio of 1:1.5 was performed for $24 \mathrm{~h}$ in six-well plates using transwell inserts (Corning, Madrid, Spain) to ensure spatial separation. NRK 52e cells (bottom) were treated with colchicine for $24 \mathrm{~h}$ and washed twice with PBS prior to co-culture with primary rat bone marrow-derived macrophages (BMDM) seeded in the insert of the transwell system [15]. At the end of the culture, cells were processed separately for gene expression determination by real-time qRT-PCR, and supernatants were collected to assess protein release by ELISA.

\section{Proliferation assay}

Proliferation was measured using the RTCA DP xCELLigence instrument (Roche Applied Science, Mannheim, Germany) as previously described [17]. Initially, a background measurement of the detector containing an E-plate insert was performed using $50 \mu \mathrm{l}$ serum-free media 
incubated for $30 \mathrm{~min}$ in the incubator $\left(37^{\circ} \mathrm{C}, 5 \% \mathrm{CO}_{2}\right)$. In parallel, NRK-52e cells were detached, quantified, and added to the insert in $100 \mu \mathrm{l}$ full growth media $(10,000$ cells per well) or treated as indicated. Proliferation was measured as an increase in impedance continuously for a period of $96 \mathrm{~h}$. The electronic readout of cell-sensor impedance is displayed in real-time as Cell Index, a value directly influenced by cell proliferation. The Cell Index value at each time point is defined and the background impedance of the well is subtracted. Data are presented as the normalized cell index as a measure for the time-dependent changes in impedance. The RTCA Software 1.2 (Omni Life Science, Bremen, Germany) was used for both data acquisition and analysis.

\section{RNA extraction and quantitative real-time polymerase chain reaction (qRT-PCR)}

Total RNA from cells was isolated using the RNeasy mini kit following the manufacturer's protocol (Qiagen, Barcelona, Spain). RNA concentrations were calculated from $\mathrm{A}_{260}$ determinations using a Nanodrop ND-1000 (NanoDrop Technologies, Wilmington, DE, USA). cDNA was synthesized by using the iScript cDNA synthesis Kit from Bio-Rad according to the manufacturer's recommendations. Quantitative RT-PCRs were performed on a Bio-Rad iCycler iQ Real-Time-PCR detection system using SYBR Green RT-PCR detection Kit (Bio-Rad, Madrid, Spain) according to the manufacturer's instructions. Sequences of specific primers are listed in Table 1. Real-time PCR results were quantified using Gene Expression Macro (version 1.1) from Bio-Rad, with glyceraldehyde-3-phosphate dehydrogenase $($ Gapdh) as internal control for constitutive expression (housekeeping gene).

\section{ELISA}

Supernatants were collected and clarified by centrifugation. A total of $50 \mu \mathrm{l}$ of each sample were applied to an ELISA 96-well-plate previously covered with a detection antimouse Lipocalin-2/NGAL monoclonal antibody (R\&D) and blocked for $1 \mathrm{~h}$. After sample incubation, biotinylated anti-mouse Lipocalin-2/NGAL detection antibody (R\&D) was added. Afterwards, HRP-conjugated avidin (Invitrogen) was added for $1 \mathrm{~h}$. Finally, colour reagent (OPD tablets, Dako, Glostrup, Denmark) was added and colour development was assessed by a microplate reader. Total protein amount in the sample was determined by the Lowry method for calculating the Lcn-2 amount per mg of total protein.

For cytokine levels of TNF- $\alpha$ and IL-10, supernatants were processed according to manufacturer's kit instructions (Endogen, Pierce Biotechnology, Barcelona, Spain).

\section{Small interfering RNA (siRNA) transfections}

siRNA transfection was performed as described previously [9]. Briefly, siRNA oligonucleotides were delivered to primary macrophages using Lipofectamine 2000 according to manufacturer's recommended protocol (Invitrogen). We used three different siRNA clones to test the knockdown efficiency. For further experiments, we applied siRNA clone 3. Lcn-2 and megalin gene expression were measured by real-time qRT-PCR.

\section{Adenoviral vectors and dose-response assay}

Adenoviral vectors were applied in order to transduce macrophages for overexpression of Lcn-2. Adenoviral control vectors for $\beta$-galactosidase (Ad $\beta$-gal) were kindly provided by Dr David Kluth and Dr Jeremy Hughes (MRC Centre for Inflammation Research, The Queen's Medical Research Institute, Edinburgh, UK). The adenoviral vector carrying cDNA encoding recombinant GFP-tagged Lcn-2 was elaborated, amplified, and purified by ViraQuest, Inc (North Liberty, IA, USA). For a dose-response assay, cells were collected, counted and aliquoted to obtain $1 \times 10^{6}$ cells, transduced with lipofectamine, and the different indicated adenoviral vectors at different doses. The optimum dose (MOI100), which yields the most effective transduction, but at the same time has a reasonable balance between cytopathic effects of the virus and its efficiency, was chosen for further experiments. Cells were incubated for $48 \mathrm{~h}$ at $37^{\circ} \mathrm{C}$ in a $\mathrm{CO}_{2}$ incubator.

\section{Phalloidin-staining}

Changes in the cytoskeleton distribution and cellular integrity were visualized by staining of F-actin stress fibbers with phalloidin as previously described [18]. Briefly, cells were fixed in $4 \%$ buffered formaldehyde, blocked, and incubated with Alexa Fluor 568-phalloidin (Molecular Probes, Darmstadt, Germany) in PBS with $1 \%$ BSA for $30 \mathrm{~min}$. Slides were washed three times with PBS and mounted using mowiol (Calbiochem, La Jolla, CA, USA). Confocal images were taken with a Leica TCS NT laser microscope (Leica Microsystems, Wetzlar, Germany) equipped with a PT-APO objective (×63).

\section{Cell migration assay}

For scratch wound assays, $1 \times 10^{5}$ NRK-52e cells were seeded in 24-well culture plates. After $24 \mathrm{~h}$, cells were starved for $16 \mathrm{~h}$ before the scratch was initiated with a small pipette tip. Detached cells were removed by washing with PBS. Stimulation was performed with CM of siMACS or scMACS for $24 \mathrm{~h}$. Recombinant Lcn-2 protein $(1 \mu \mathrm{g} / \mathrm{ml})$ 
Table 1 Primer sequences used in this study

\begin{tabular}{|c|c|c|}
\hline Gene (accession number) & Source & Primer sequence \\
\hline $\mathrm{Rn} \_L c n-2$ & Invitrogen & Fw: 5'-CAAGTGGCCGACACTGACTA-3' \\
\hline NM_008491 & & Rvs: 5'-GGTGGGAACAGAGAAAACGA-3' \\
\hline $\mathrm{Rn} \_$Gapdh & Invitrogen & Fw: 5'-CCGCCAATGTATCCGTTGTG-3' \\
\hline NM_017008 & & Rvs: 5'-TAGCCCAGGATGCCCTTTAGT-3' \\
\hline $\mathrm{Rn} \_K i-67$ & Invitrogen & Fw: 5'-AGACGTGACTGGTTCCCAAC-3' \\
\hline XM_00105622 & & Rvs: 5'-ACTGCTTCCCGAGAACTGAA-3' \\
\hline $\mathrm{Rn} \_P c n a$ & Invitrogen & Fw: 5'-AGGACGGGGTGAAGTTTTCT-3' \\
\hline NM_022381 & & Rvs: 5'-CAGTGGAGTGGCTTTTGTGA-3' \\
\hline $\mathrm{Rn} \_$E-cadherin & Invitrogen & Fw: 5'-GGTCTCTTGTCCCTTCCACA-3' \\
\hline NM_031334 & & Rvs: 5'-CTCCAGACCCACACCAAAGT-3' \\
\hline $\mathrm{Rn} \_$Vimentin & Invitrogen & Fw: 5'-СТCTTCСАAАCTTTТССТССС-3' \\
\hline NM_031140 & & Rvs: 5'-AGTTTCGTTGATAACCTGTCC-3' \\
\hline $\mathrm{Rn} \_$Aquaporin-1 & Invitrogen & Fw: 5'-AAGTCCCCCTCACTCCAAAG-3' \\
\hline NM_012778.1 & & Rvs: 5'-GGGACTTCCTCTCCCTCAAA-3' \\
\hline $\mathrm{Rn} \_$Aquaporin-2 & Invitrogen & Fw: 5'-TGTCTCCTTCCTTCGAGCTG-3' \\
\hline NM_012909 & & Rvs: 5'-AGGTCCCCACGGATTTCTAC-3' \\
\hline Rn_Cpt-1 XM_006531658.3 & Invitrogen & $\begin{array}{l}\text { Fw: 5'-TATGTGAGGATGCTGCTTCC-3' } \\
\text { Rvs: 5'-CTCGGAGAGCTAAGCTTGTC-3' }\end{array}$ \\
\hline $\begin{array}{l}\text { Rn_Pdk4 } \\
\text { NM_001163487.1 }\end{array}$ & Invitrogen & $\begin{array}{l}\text { Fw: 5'-GGATTACTGACCGCCTCTTTAGTT-3' } \\
\text { Rvs: 5'-GCATTCCGTGAATTGTCCATC-3' }\end{array}$ \\
\hline $\begin{array}{l}\mathrm{Rn} \_ \text {Megalin } \\
\text { NM_001017443 }\end{array}$ & Qiagen & Quantitect primer assay \\
\hline $\begin{array}{l}\mathrm{Rn} \_L c n-2 R \\
\mathrm{NM} \_177421\end{array}$ & Qiagen & Quantitect primer assay \\
\hline $\begin{array}{l}\text { Rn_Ppard } \\
\text { NM_013196 }\end{array}$ & Qiagen & Quantitect primer assay \\
\hline 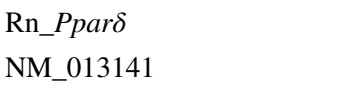 & Qiagen & Quantitect primer assay \\
\hline $\begin{array}{l}\mathrm{Rn} \_ \text {Ppary } \\
\mathrm{NM} \_001145366\end{array}$ & Qiagen & Quantitect primer assay \\
\hline $\begin{array}{l}\mathrm{Rn} \_ \text {Fabp4 } \\
\mathrm{NM} \text { _053365 }\end{array}$ & Qiagen & Quantitect primer assay \\
\hline
\end{tabular}

was used as positive control. Images were acquired using an Axio-Scope microscope (Carl Zeiss Microimaging, Jena, Germany) and quantification of the scratch area was followed using Image $\mathrm{J}$ software. The $0 \mathrm{~h}$ value was normalized to the $24 \mathrm{~h}$ value of each group. The relative migration rate defines the mean value of at least three independent experiments using triplicates for each condition.

\section{Immunostaining}

Cells were fixed in $3.7 \%$ paraformaldehyde at $37^{\circ} \mathrm{C}$ and subsequently permeabilized with aceton on ice. After washing, cells were blocked with goat serum (Sigma, Madrid, Spain) for $1 \mathrm{~h}$ and primary antibody was added to detect the proliferation marker PCNA (Santa Cruz Biotechnology, Heidelberg, Germany) and KI-67 (Abcam,
Cambridge, UK). Sections were mounted with mowiol (Calbiochem). Images were obtained with an Axio-scope 40 and documented with AxioVision software (Zeiss). Negative controls, lacking primary antibodies, showed no staining. Antibodies to detect E-cadherin were purchased from Santa Cruz Biotechnology (Heidelberg, Germany), Vimentin from Sigma (Madrid, Spain), cytokeratin-18 from Millipore (Madrid, Spain) and $\beta$-catenin from BD Bioscience (Madrid, Spain).

\section{Lcn-2 receptor binding and GFP-staining}

For Lcn-2 receptor studies, NRK-52e cells were seeded in a six-well-plate at a density of $1 \times 10^{6}$ cells per well and incubated with CM from macrophages overexpressing GFPtagged Lcn-2 $(1 \mu \mathrm{g} / \mathrm{ml})$ for $24 \mathrm{~h}$ in the presence or absence of 

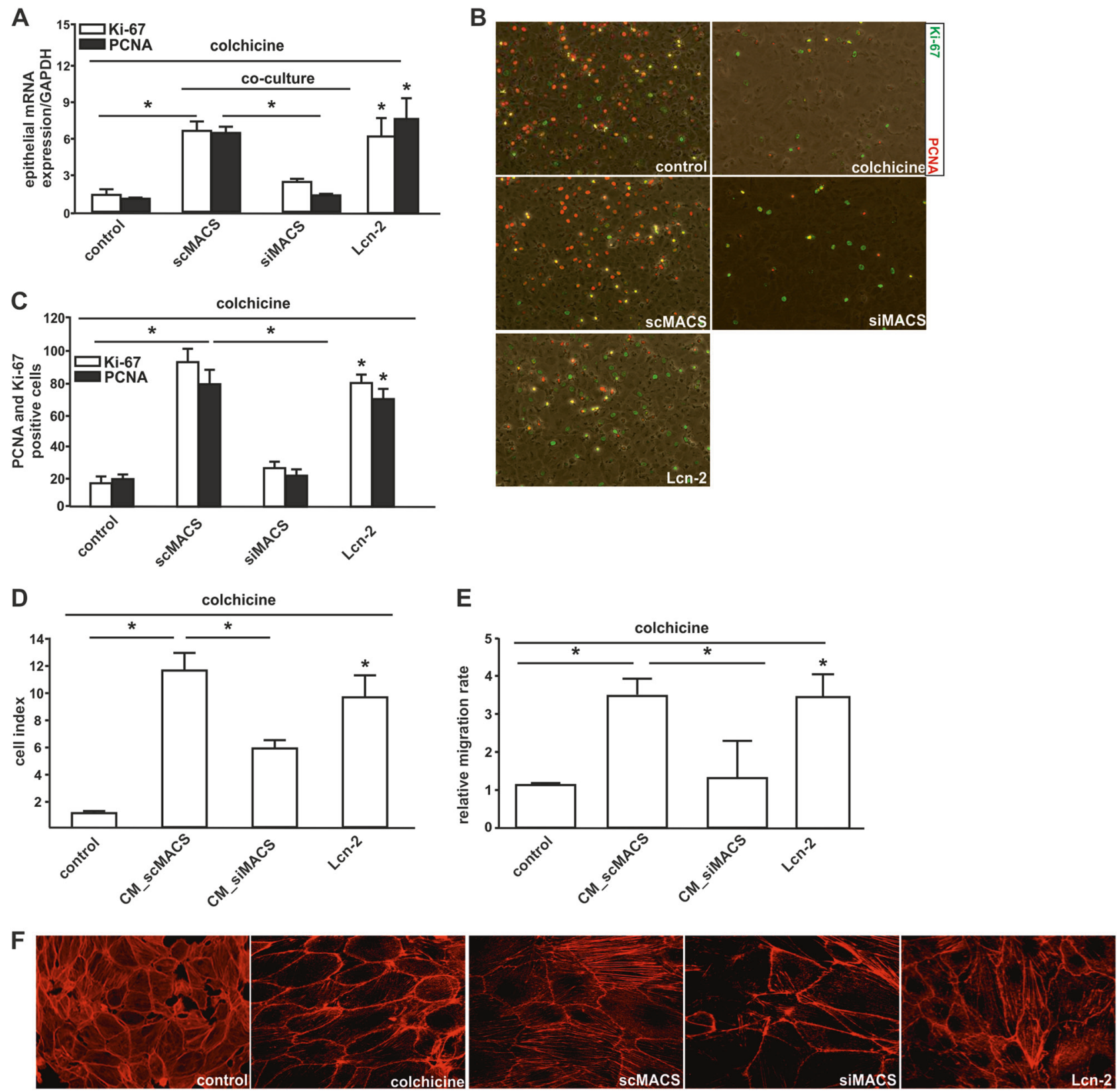

Fig. 1 Macrophage-derived Lcn-2 recues epithelial cell proliferation. a-c NRK52e cells were treated with colchicine to induce cell cycle arrest and were either co-cultured with rat BMDM (scMACS or siMACS) in a Transwell system for $24 \mathrm{~h}$ or left untreated (control). Recombinant Lcn-2 was used as positive control at a concentration of $1 \mu \mathrm{g} / \mathrm{ml}$ for $24 \mathrm{~h}$. a mRNA expression by qRT-PCR of the proliferation markers Pcna and $K i-67$ was determined. $n=6$. Data are means \pm S.E. M, * $p \leq 0.05$. b Immunofluorescence staining of PCNA (red) and Ki-67 (green) was performed to confirm the induction of cellular proliferation in co-cultured renal epithelial cells. Representative pictures from six independent experiments are shown (magnification $\times 20$ ) in untreated cells (control), following colchicine treatment, colchicine-treated cells in co-culture with scMACS or with siMACS or with recombinant Lcn2 protein. c Quantification of Ki-67 and PCNA positive proliferating cells was performed using Image J. $n=6$, data are means \pm S.E.M.,
$* p \leq 0.05$. d Proliferation in cells treated with conditioned medium (CM) of the different experimental settings was analysed using xCELLigence for $96 \mathrm{~h}$. Results were calculated as cell index. Six independent experiments (in triplicates) were performed and analysed as mean data \pm S.E.M, $* p \leq 0.05$. e Migration was quantified by scratch-wound-assay in NRK52e cells treated with CM for $24 \mathrm{~h}$. Relative migration rate was calculated as described in Materials and Methods. $n=6$ (in triplicates), data are means \pm S.E.M, ${ }^{*} p \leq 0.05$. f Cellular cytoskeleton distribution was measured by staining for Factin fibres using Phalloidin. Representative pictures from six independent experiments are shown (magnification $\times 60$ ) in untreated cells (control), following colchicine treatment, in colchicine-treated cells in co-culture with scMACS, in colchicine-treated cells in co-culture with scMACS, and in colchicine-treated cells stimulated with recombinant Len-2 
the competing recombinant Lcn-2 without GFP-tag in excess $(50 \mu \mathrm{g} / \mathrm{ml})$. As control, CM from macrophages transduced with the control vector containing $\beta$-galactosidase was used. At the end of incubation, cells were prepared for GFPdetection via immunofluorescence. Therefore, cells were washed with PBS, fixed in $3.7 \%$ paraformaldehyde, and permeabilized with acetone on ice. Cells were blocked for 1 $\mathrm{h}$ using goat serum (Sigma) and were incubated with a rabbit monoclonal anti-GFP antibody (kindly provided by Dr. S. Pons, IIBB-CSIC-IDIBAPS, Barcelona, Spain) for $2 \mathrm{~h}$ at room temperature, followed by the incubation with the secondary antibody for $1 \mathrm{~h}$ (rabbit anti-goat IgG conjugated with Alexa Fluor 488). Cells were mounted with mowiol (Calbiochem), and images were obtained with an Axioscope 40 and documented with AxioVision software (Zeiss).

\section{Iron determination}

NRK-52e cells were left untreated or were stimulated either with $\mathrm{CM}$ from control macrophages (CM $\beta$-gal-MACS) or CM from Lcn-2-overexpressing macrophages (CM Lcn-2-MACS), both supplemented with $20 \mathrm{mg} / \mathrm{ml}$ iron (FERIV, G.E.S. Genericos españoles laboratorio, S.A., Madrid, Spain). Iron content was measured using an ADVIA 2400 (Siemens Medical Diagnostics, Eschborn, Germany) multichannel analyser.

\section{SDS-PAGE and western blot analysis}

For western blot analysis, cells were scraped off, centrifuged, resuspended in lysis buffer (urea $7 \mathrm{M}$, thiourea 2 M, CHAPS $4 \%$, Tris $40 \mathrm{mM}$, PMSF, DTT), and sonicated for $1 \mathrm{~min}$. Subsequently, protein concentrations were determined with the Bradford method using a commercial kit from Bio-Rad. Equal amounts of protein were resolved on SDS polyacrylamide gels and blotted onto nitrocellulose membranes. Unspecific binding was blocked with 5\% milk in $0.6 \%$ Tween-TBS (TTBS) for $1 \mathrm{~h}$. Primary antibody (Anti-pAkt: Cell Signalling, Frankfurt, Germany) was added in TTBS $/ 5 \%$ dry milk and incubated overnight at $4^{\circ}$ C. For protein detection, blots were incubated with the respective secondary antibody in TTBS/5\% dry milk, followed by ECL-detection of specific proteins using the Quantity One imaging system (Bio-Rad). Equal protein loading was determined by visual detection by staining the membranes with Ponceau S and actin (Sigma) as a loading control. Moreover, total Akt (Cell Signalling) expression was quantified respectively as controls.

\section{Statistical analysis}

Each experiment was performed at least five times. The $p$ values were calculated using One-way ANOVA and considered significant at $* p<0.05$.

\section{Results}

\section{Macrophage-derived Lcn-2 reverses epithelial cell cycle arrest}

To study the impact of macrophage-derived Lcn-2 on epithelial cell proliferation, we used a transient siRNA approach to knockdown Lcn-2 in bone marrow-derived macrophages (BMDM). Supplementary figure 1 shows the knockdown efficiency of three different siRNA clones, all of which achieved $\sim 70 \%$ reduction in protein expression. Knockdown of Len-2 did not compromise macrophage viability but significantly reduced the expression of IL-10 and increased the induction of TNF- $\alpha$.

We applied a co-culture model of rat BMDM and NRK52e rat renal epithelial tubular cells, as previously established by our group [14]. In order to induce cell cycle arrest in epithelial cells, we stimulated cells with colchicine for $24 \mathrm{~h}$, which blocks the G2- to M-phase transition and subsequently reduces cell proliferation. After colchicine exposure, epithelial cells were washed and co-cultured in a transwell system with BMDMs treated with scrambled siRNA (scMACS), a specific Lcn-2-siRNA (siMACS), Lcn2 protein (Lcn-2) or left untreated (control). Results in Fig. 1a showed a significant increase in mRNA expression of the proliferation markers $\mathrm{Ki}-67$ and Pcna after co-culture of epithelial cells with scMACS; whereas, the knockdown of Lcn-2 in macrophages inhibited the expression of the epithelial cell proliferation markers. Stimulation with authentic Lcn-2 protein significantly enhanced renal epithelial cell proliferation. This response is in a dosedependent manner (Suppl. Fig. S3). Recombinant Lcn-2 at a concentration of $1 \mu \mathrm{g} / \mathrm{ml}$ was used for the experiments of this study.

Immunofluorescent staining of Ki-67 (green) and PCNA (red) (Fig. 1b) with quantification of positive cells using Image J software (Fig. 1c) corroborated qRT-PCR-based observations. Colchicine-treated epithelial cells are poorly proliferating, whereas the co-culture of epithelial cells with scMACS as well as the treatment with authentic Lcn-2 promotes the re-entry of epithelial cells in an active cell cycle, which is represented by enhanced expression of both proliferation markers $\mathrm{Ki}-67$ and PCNA. In contrast, renal epithelial cells expressed significantly less Ki-67 and PCNA after their co-culture with siLcn-2 MACS, indicating that the cell cycle arrest remained intact.

We used the supernatant of the different experimental settings to test Lcn-2 in real-time cellular proliferation using xCELLigence. Results are represented as cell index, and showed a significantly enhanced proliferation after stimulation with the conditioned medium of scMACS that contained Lcn-2, whereas CM_siLcn-2 MACS significantly reversed this effect (Fig. 1d). Recombinant Lcn-2 protein 
A
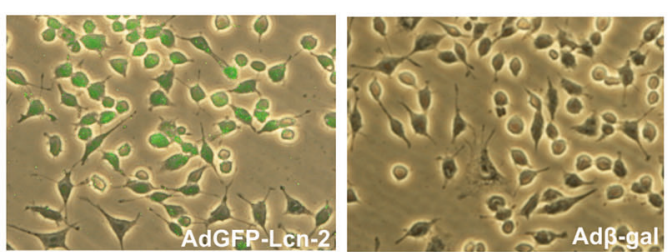

B

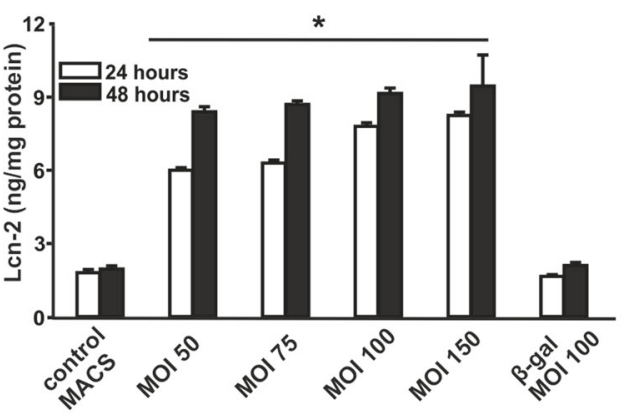

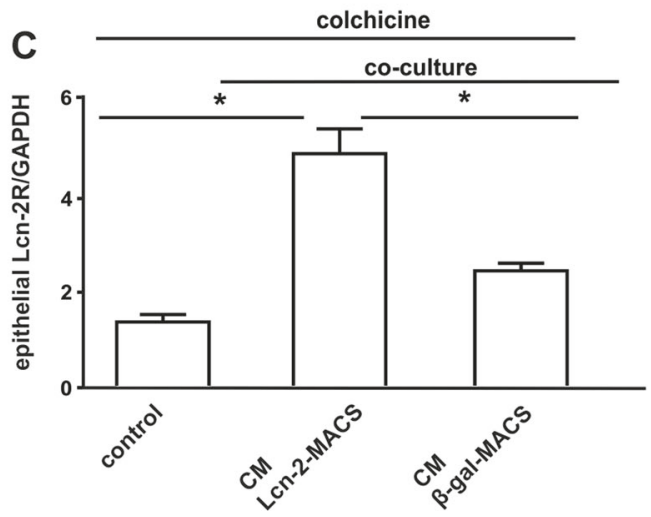

D

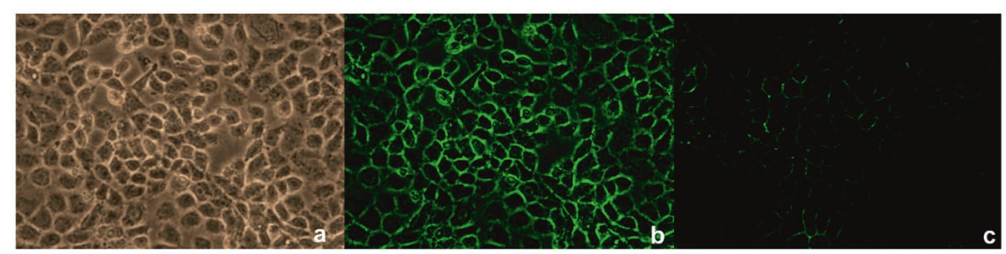

E

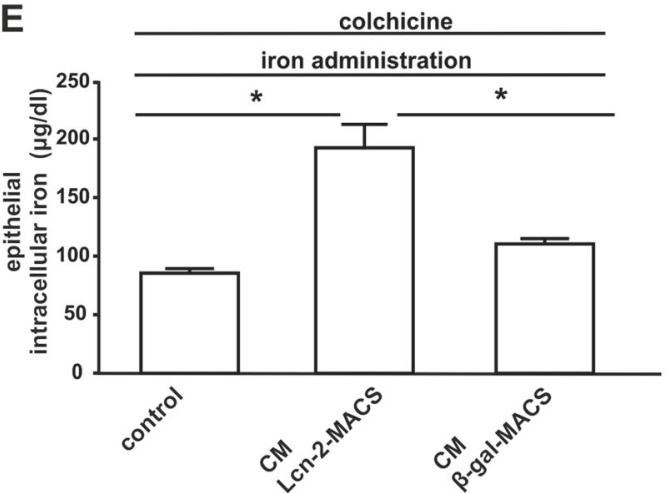

G

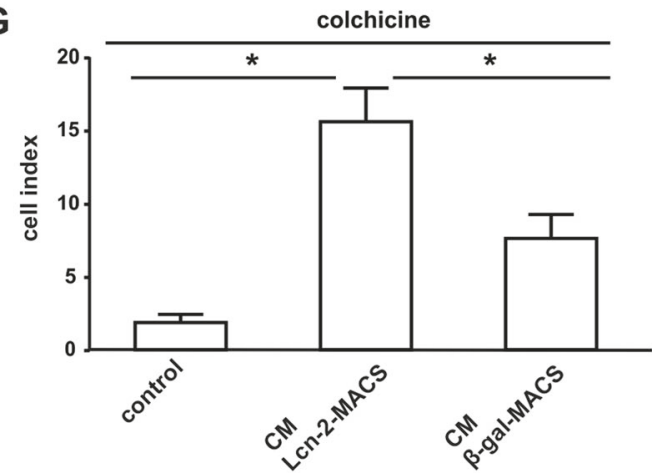

$\mathbf{F}$

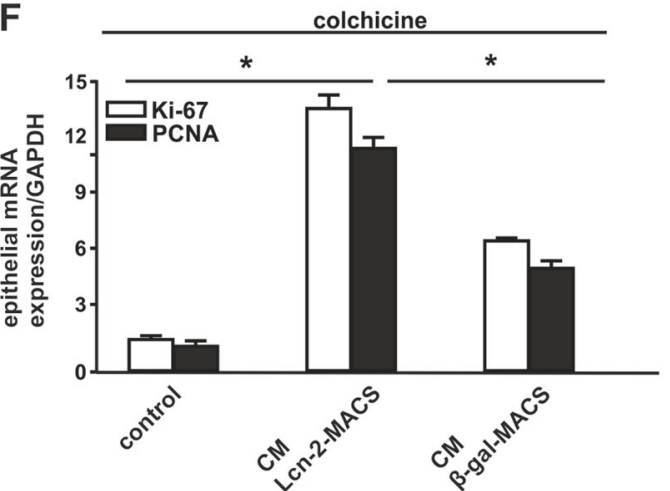

was used as positive control and corroborated its proproliferative effect on cell cycle arrested renal epithelial cells. Since epithelial repair mechanisms are not only associated with proliferation of remaining cells, but also migration of epithelial cells, we performed a scratch woundassay with colchicine-treated NRK52e cells. In order to 
Fig. 2 Macrophage-derived Lcn-2 induces cellular proliferation by binding to its specific receptor on the surface of renal epithelial cells. a GFP immunofluorescence confirmed the overexpression of Lcn-2 in BMDM (left). The control adenoviral construct encoding for $\beta$ galactosidase ( $\beta$-gal) was used as transduction control (right) (magnification $\times 40$ ). $\mathbf{b}$ Lcn- 2 protein was measured in transduced primary macrophages at different multiplicity of infection (MOI). $n=6$, data are means \pm S.E.M, ${ }^{*} p \leq 0.05$ vs. control MACS. c Induction of the specific Lcn-2 receptor, $L c n-2 R$, measured by qRT-PCR in cells treated with colchicine, without further stimulus (control) or stimulated with macrophage supernatants (CM Lcn-2-MACS and CM $\beta$-galMACS) for 24 h. $n=6$, data are means \pm S.E.M, $* p \leq 0.05$. d NRK52e cells were incubated with CM from GFP-tagged Lcn-2 overexpressing macrophages for $24 \mathrm{~h}$ (CM Lcn-2-MACS) in order to evaluate binding to its receptor. Cells were viewed on a Leica TCS NT laser microscope (magnification $\times 40$ ). Representative pictures of 6 independent experiments are shown. e NRK52e cells under cell cycle arrest were incubated with CM from Lcn-2 overexpressing macrophages and $\beta$ gal-expressing macrophages for $24 \mathrm{~h}$ and supplemented with $20 \mathrm{mg} / \mathrm{ml}$ iron. Cellular iron content significantly increased after treatment with CM from Lcn-2-MACS, indicating Len-2 functionality in providing and trafficking iron to epithelial cells. $n=6$, data are means \pm S.E.M, ${ }^{*} p \leq 0.05$. Proliferation was analysed by (f) mRNA expression of $\mathrm{Ki}$ 67 and Pcna and $\mathbf{g}$ by using xCELLigence for $96 \mathrm{~h}$. Results were calculated as normalized cell index. Six independent experiments (in triplicates) were performed and analysed as mean data \pm S.E.M, ${ }^{*} p \leq$ 0.05

decipher the role of the secreted Lcn-2, we compared the effect of conditioned medium of scMACS and siLcn-2MACS on epithelial migration. In contrast to CM_scMACS, CM_siMACS provoked a significantly reduced wound closure after $24 \mathrm{~h}$ (Fig. 1e). Again, recombinant Lcn-2 was used as positive control and showed similar results as CM_scMACS. However, since cells were stimulated for $24 \mathrm{~h}$, it is still possible that proliferation of cells adds to the observed effects.

During the phases of renal repair, not only proliferation and migration of epithelial cells are of crucial importance, but also the induction and/or maintenance of the epithelial phenotype of renal tubular cells. Therefore, we determined the integrity of F-actin fibres of the cytoskeleton in cocultured epithelial cells with phalloidin staining (Fig. 1f). Compared to untreated cells, colchicine treatment induces the depolymerisation and loss-of actin stress fibres by becoming thicker and shorter, finally accumulating in a junctional ring around the cells. In scMACS-co-cultured renal cells, the F-actin stress-fibres were long and thin, covering the cell to support and maintain its normal morphology. The treatment with Lcn-2 protein also maintains normal F-actin distribution. Epithelial cells that were cocultured with siLcn-2 MACS showed a major loss-of-stress fibres, resulting in total depolymerisation.

The response of NRK-52e cells to the cell cycle inhibitor colchicine treated in usual culture medium compared to macrophage conditioned medium (CM_MACS) is shown in Supplemental Figure S2. We compared epithelial viability by Alamar blue assay, cell number as fold induction normalized to day 0 , and the proliferation markers PCNA and Ki-67 analysed by qRT-PCR at different time points. Results reflect restored proliferation in cells cultured with CM_MACS after colchicine treatment compared to the standard medium.

\section{Receptor-mediated binding and uptake of macrophage-derived Lcn-2 restores proliferation of epithelial cells}

In order to further prove the pro-proliferative capacity of macrophage-derived Len-2 on renal epithelial cells, we generated Lcn-2-overexpressing macrophages. Therefore, we transduced a GFP-tagged Lcn-2 encoding adenoviral vector (AdLcn-2) in BMDM for stable Lcn-2 production. We checked transduction efficiency by immunofluorescent analysis of GFP-positive cells (Fig. 2a). As control, an adenoviral construct encoding $\beta$-galactosidase ( $\beta$-gal) was used (Fig. 2a, right). We verified Lcn-2 protein expression applying different multiplicity of infection (MOI) compared to transduced cells with control virus ( $\beta$-gal) (Fig. $2 b)$.

We previously described a role for the specific receptor Lcn-2R in mediating Lcn-2-dependent pro-repair capacity in vivo. Therefore, we tested the involvement of $\mathrm{Lcn}-2 \mathrm{R}$ in vitro (Fig. 2c, d). We found a significant induction of $L c n$ $2 R$ mRNA in epithelial cells upon incubation with supernatant of Lcn-2-overexpressing macrophages compared to $\beta$ gal-expressing control macrophages for $24 \mathrm{~h}$ (Fig. 2c). Fig. 2d depicts a competition assay. We incubated renal epithelial NRK52e cells with supernatant from BMDM overexpressing GFP-tagged Lcn-2 (CM Lcn-2-MACS). We deduced protein-receptor interactions, since the fluorescent signal detected on the surface of the cells was significantly reduced after further addition of an excess amount of unlabelled recombinant Lcn-2 $(50 \mu \mathrm{g} / \mathrm{ml})$, indicating competition for receptor binding on the cellular surface. Additionally, we confirmed the uptake of Lcn-2 by its function of iron trafficking (Fig. 2e); we pre-incubated supernatant from $\beta$-galMACS and Lcn-2-MACS with $20 \mathrm{mg} / \mathrm{ml}$ iron prior to the stimulation of renal epithelial cells. Iron content of the cellular lysates showed a significant increase in intracellular iron concentration upon stimulation with conditioned medium from Lcn-2-MACS, thus indicating that the macrophage-secreted protein is functional. In order to prove the growth-promoting effect of macrophage-released Lcn-2 on cellular proliferation, we measured mRNA detection of $\mathrm{Ki}-67$ and Pcna (Fig. 2f) as well as applying xCELLigence real-time proliferation measurements (Fig. $2 \mathrm{~g}$ ). Supernatants of Lcn-2-overexpressing macrophages significantly enhanced cell proliferation of NRK52e cells compared to stimulation of epithelial cells with supernatant of $\beta$-gal control macrophages. 

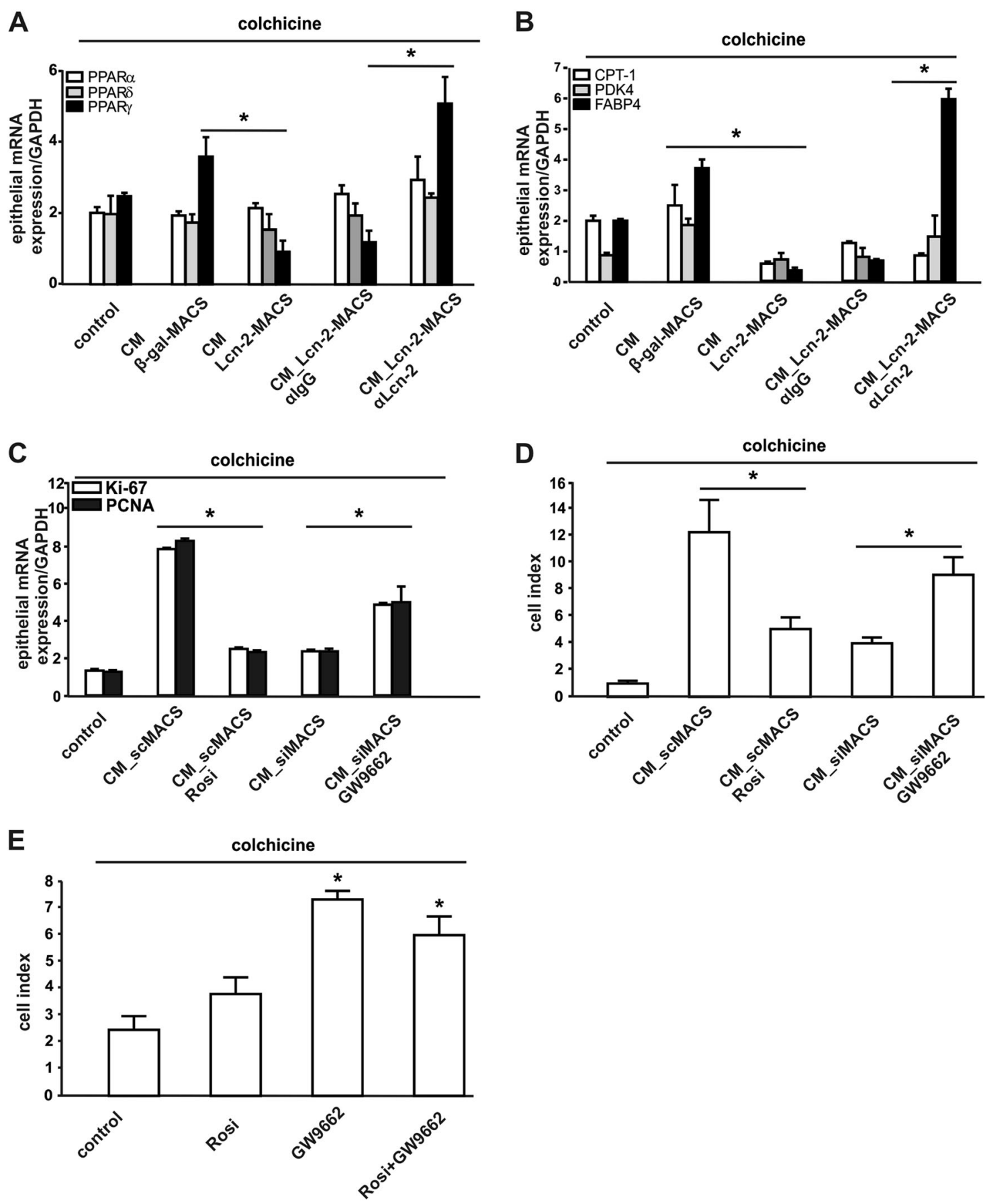

Fig. 3 Lcn- 2 promotes epithelial cell proliferation by PPAR $\gamma$ inhibition. a-b NRK52e cells were treated with colchicine to induce cell cycle arrest. Then, cells were either left without further stimulus (control) or stimulated with $\mathrm{CM}$ from control-transfected $\beta$-gal expressing macrophages ( $\beta$-gal MACS) or Lcn-2 overexpressing BMDM (Lcn-2-MACS) in the presence of a Lcn-2 neutralizing antibody $(\alpha \mathrm{Lcn}-2 ; 3.5 \mu \mathrm{g} / \mathrm{ml})$ or an isotype matching IgG control antibody $(\alpha \mathrm{IgG}, 3.5 \mu \mathrm{g} / \mathrm{ml})$. a mRNA of Ppar $\alpha, \gamma$, and $\delta$ as well as (b) PPAR $\alpha$ target gene $C p t-1$, PPAR $\delta$ target gene $P d k 4$, and PPAR $\gamma$ target gene Fabp 4 were measured at mRNA level using qRT-PCR. $n=6$, data are means \pm S.E.M., ${ }^{*} p \leq 0.05$. c The PPAR $\gamma$ agonist rosiglitazone $(1 \mu \mathrm{M})$ was added to CM containing Lcn-2 (CM_scMACS) and the PPAR $\gamma$ antagonist GW9662 (100 nM) was supplemented to CM from Lcn-2

knockdown macrophages (CM_siMACS) and proliferative markers $P c n a$ and $K i-67$ were measured. $n=6$, data are means \pm S.E.M., ${ }^{*} p \leq$ 0.05 . d Proliferation was analysed using xCELLigence for $96 \mathrm{~h}$ and results were calculated as cell index. Five independent experiments (in triplicates) were performed and analysed as mean data \pm S.E.M., $* p \leq$ 0.05 . e NRK52e cells were treated with colchicine and were stimulated either with the PPAR $\gamma$ agonist rosiglitazone $(1 \mu \mathrm{M})$ or the PPAR $\gamma$ antagonist GW9662 (100 nM) alone or in combination or remained untreated (control). Proliferation was analysed using xCELLigence for $96 \mathrm{~h}$ and results were calculated as cell index. Six independent experiments (in triplicates) were performed and analysed as mean data \pm S.E.M., ${ }^{*} p \leq 0.05$ 
A
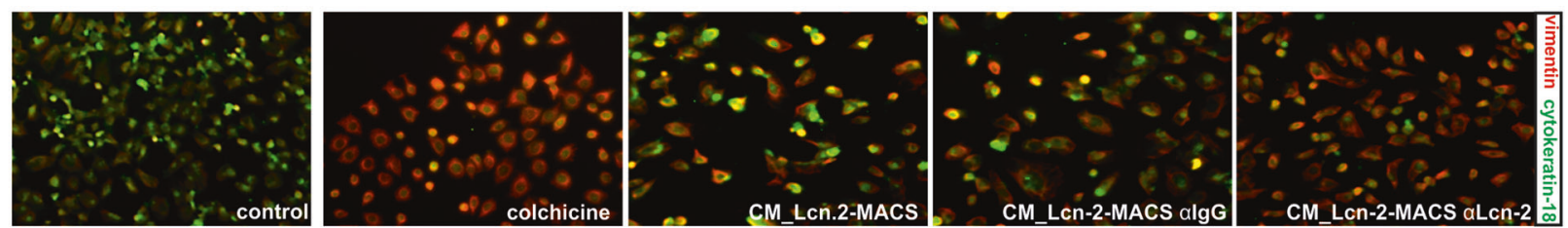

B

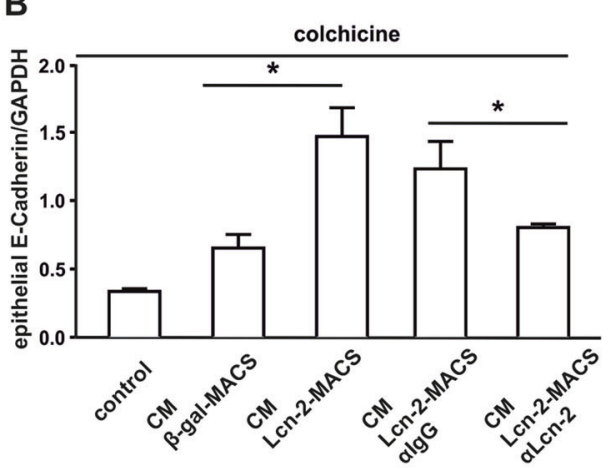

D

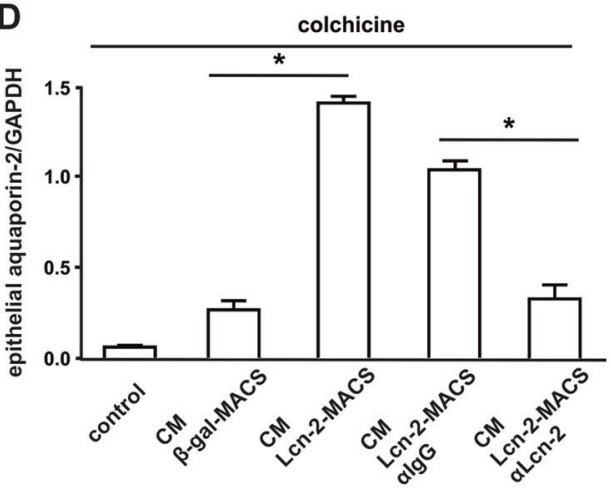

$\mathbf{F}$

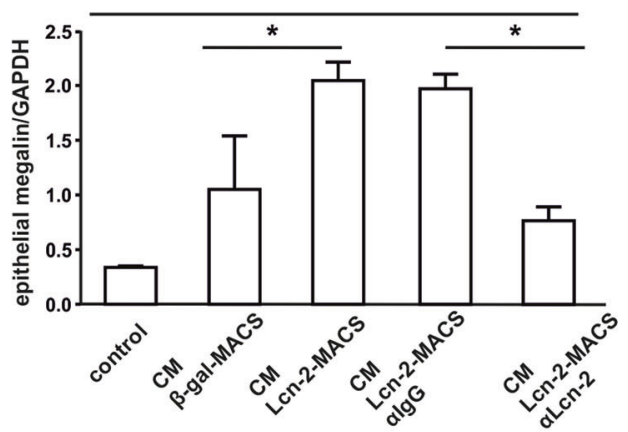

Fig. 4 Lcn-2 induces the expression of epithelial markers in damaged renal epithelial cells. a Immunofluorescence staining for the mesenchymal marker vimentin (red) and the epithelial marker cytokeratin-18 (green) in untreated cells, colchicine-treated cells colchicine-treated cells stimulated with CM from Lcn-2 overexpressing BMDM (Lcn-2-MACS), colchicine-treated cells stimulated with CM from Lcn-2 overexpressing BMDM (Lcn-2-MACS) in the presence of an IgG control antibody, and colchicine-treated cells stimulated with CM from Lcn-2 overexpressing BMDM (Lcn-2-MACS) in the presence of the Lcn-2 neutralizing antibody. Representative pictures of six independent experiments are shown for each condition. b-f NRK-52e cells were treated with colchicine to induce cell cycle arrest. Afterwards, cells were either left without further stimulus (control) or stimulated with $\mathrm{CM}$ from $\beta$-gal-expressing macrophages ( $\beta$-gal MACS) or Lcn-2 overexpressing BMDM (Lcn-2-MACS) in the presence of a neutralizing antibody against Lcn-2 ( $\alpha \mathrm{Lcn}-2 ; 3.5 \mu \mathrm{g} /$ $\mathrm{ml})$ or an isotype matching $\operatorname{IgG}$ control antibody $(\alpha \mathrm{IgG}, 3.5 \mu \mathrm{g} / \mathrm{ml})$. mRNA expression of $\mathbf{b}$ E-cadherin, (c) aquaporin 1, and $\mathbf{d}$ aquaporin 2 was determined after $24 \mathrm{~h}$ of $\mathrm{CM}$ treatment. e Basal aquaporin 1 and aquaporin 2 mRNA expression in control NRK-52e cells (f) megalin mRNA was determined after $24 \mathrm{~h}$ of CM treatment by qRT-PCR, $n=6$, data are means \pm S.E.M., ${ }^{*} p \leq 0.05$ 


\section{Len-2 promotes epithelial cell proliferation by inhibiting PPARY activation}

In order to decipher the mechanism of Lcn-2-induced epithelial proliferation, we focused on PPAR-dependent pathways, since Lcn-2 has previously been described as a selective modulator of PPAR $\gamma$ activation and function [19]. Therefore, we first determined the expression of PPARs in epithelial cells stimulated with the supernatant (CM) of Lcn-2-overexpressing BMDMs in the presence or absence of a neutralizing antibody against Lcn-2, compared to an isotype matched $\operatorname{IgG}$ control antibody (Fig. 3a). Results show that the expression of $P P A R \gamma$, but not PPAR $\alpha$ or $P P A R \delta$ was modulated by Lcn-2. CM of Lcn-2-MACS induced a significant reduction in PPAR expression, whereas neutralization of Lcn-2 significantly induced PPAR . Analysis of PPAR-target genes (Fig. 3b) demonstrate that the PPAR $\delta$ target gene Pdk4, the PPAR $\gamma$ target gene Fabp4, and the Ppara target gene Cpt-1 were significantly reduced after stimulation with conditioned medium from Lcn-2 overexpressing macrophages. The addition of a neutralizing Lcn-2 antibody only reversed the effect on the PPAR $\gamma$ target gene Fabp4, whereas Cpt-1 and Pdk4 remained unaltered. This indicates a Lcn-2-dependent selectivity for Ppary.

To further study the role of Lcn-2-dependent Ppary inhibition regarding cellular proliferation, we determined mRNA expression of the Lcn-2-induced proliferation markers Ki-67 and Pcna in the presence of the PPAR $\gamma$ agonist rosiglitazone $(1 \mu \mathrm{M})$. In comparison, we treated epithelial cells with supernatant from siLcn-2 MACS supplemented with the PPAR $\gamma$ antagonist GW9662 (100 nM) (Fig. 3c). Stimulation with supernatant of scMACS induced cellular proliferation; whereas, the addition of the PPAR $\gamma$ agonist rosiglitazone significantly blocked this effect. Medium from siMACS reduced cellular proliferation, while supplementation of the PPAR $\gamma$ antagonist GW9662 allowed recovered proliferation. Furthermore, functional proliferation analysis using real-time measurements applying xCELLigence supported these findings (Fig. 3d). Inhibition of the PPAR $\gamma$ pathway using GW9662-induced cellular proliferation; whereas, the addition of rosiglitazone alone remained without effect (Fig. 3e).

\section{Lcn-2 promotes the expression of epithelial markers in damaged cells}

Next, we checked if cells re-express epithelial markers in the presence of Lcn-2. Immunofluorescence staining for vimentin (red) and cytokeratin-18 (green) (Fig. 4a) show that incubation with Lcn-2-containing conditioned media led to a significant increase in fluorescent signal for the epithelial marker cytokeratin-18. In contrast, vimentin staining was increased in cells, where Lcn-2 was absent or neutralized.

The mRNA expression of the epithelial markers Ecadherin (Fig. 4b) and aquaporines 1 and 2 (Fig. 4c, d) also corroborated the positive correlation with Lcn-2 presence. In the kidney, the expression of aquaporins is a typical feature of epithelial phenotype. NRK52e cells are a commonly used proximal tubule cell line expressing characteristic markers, such as aquaporin 1. However, our results demonstrate that these cells also express some characteristics of non-proximal tubule origin, such as aquaporin 2, but to a significantly lower extent (Fig. 4e).

Taking into consideration that the expression of the multi-ligand, endocytic receptor megalin was previously associated to epithelium, further considering that megalin was described as an unspecific, alternative receptor for Lcn2 in the kidney, we next investigated if Lcn-2 is able to induce the expression of megalin in vitro. We detected a significant increase in megalin mRNA expression in renal epithelial cells incubated with Lcn-2-containing macrophage supernatant, whereas co-treatment with a neutralizing Lcn-2 antibody reversed this effect (Fig. 4f).

\section{The PI3K-Akt-pathway and megalin cooperate with Lcn-2 to induce proliferation and to preserve an epithelial phenotype}

Considering that megalin might act as unspecific receptor to promote Lcn-2-induced pro-proliferative effects, we further focused on the activation of megalin downstream signalling pathways in response to Lcn-2. We determined enhanced Akt phosphorylation via western blot in epithelial cells stimulated with supernatant of Lcn-2 overexpressing macrophages for $24 \mathrm{~h}$ (Fig. 5a), whereas neutralizing Lcn-2 inhibited this effect. Interestingly, the knockdown of megalin in epithelial cells inhibited Lcn-2-induced Aktphosphorylation, suggesting megalin as an additional receptor for Lcn-2, as previously proposed. Megalin knockdown efficiency is shown in supplemental figure S4. As a positive control of the contribution of the Akt-pathway, we added the known PI3K-Akt-pathway inhibitor LY294002 $(10 \mu \mathrm{M})$ to Lcn-2 containing supernatants. Total Akt was measured as loading control and was not modified in any of the used conditions. Furthermore, Lcn-2dependent effects on E-cadherin (Fig. 5b) and vimentin (Fig. 5c) mRNA expression were evaluated in renal epithelial cells with a megalin knockdown and in LY294002treated cells. Both the knockdown of megalin and inhibition of the PI3K-Akt-pathway significantly reversed the effects of Lcn-2 on E-cadherin as well as and vimentin. In order to establish that the PI3K-Akt-pathway is implicated in promoting the growth-favouring effect of macrophage-derived Lcn-2, we tested the proliferative outcome using mRNA 

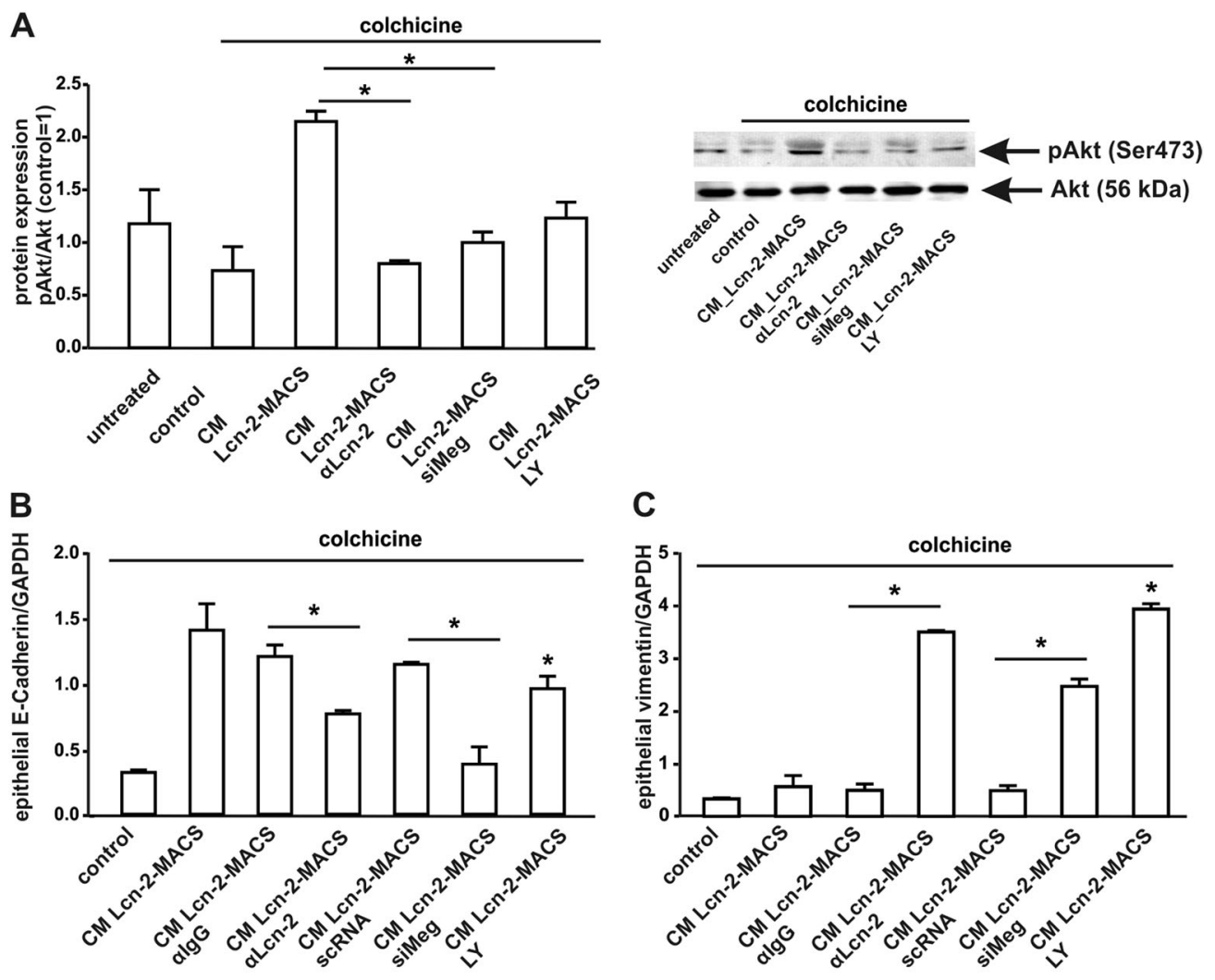

C
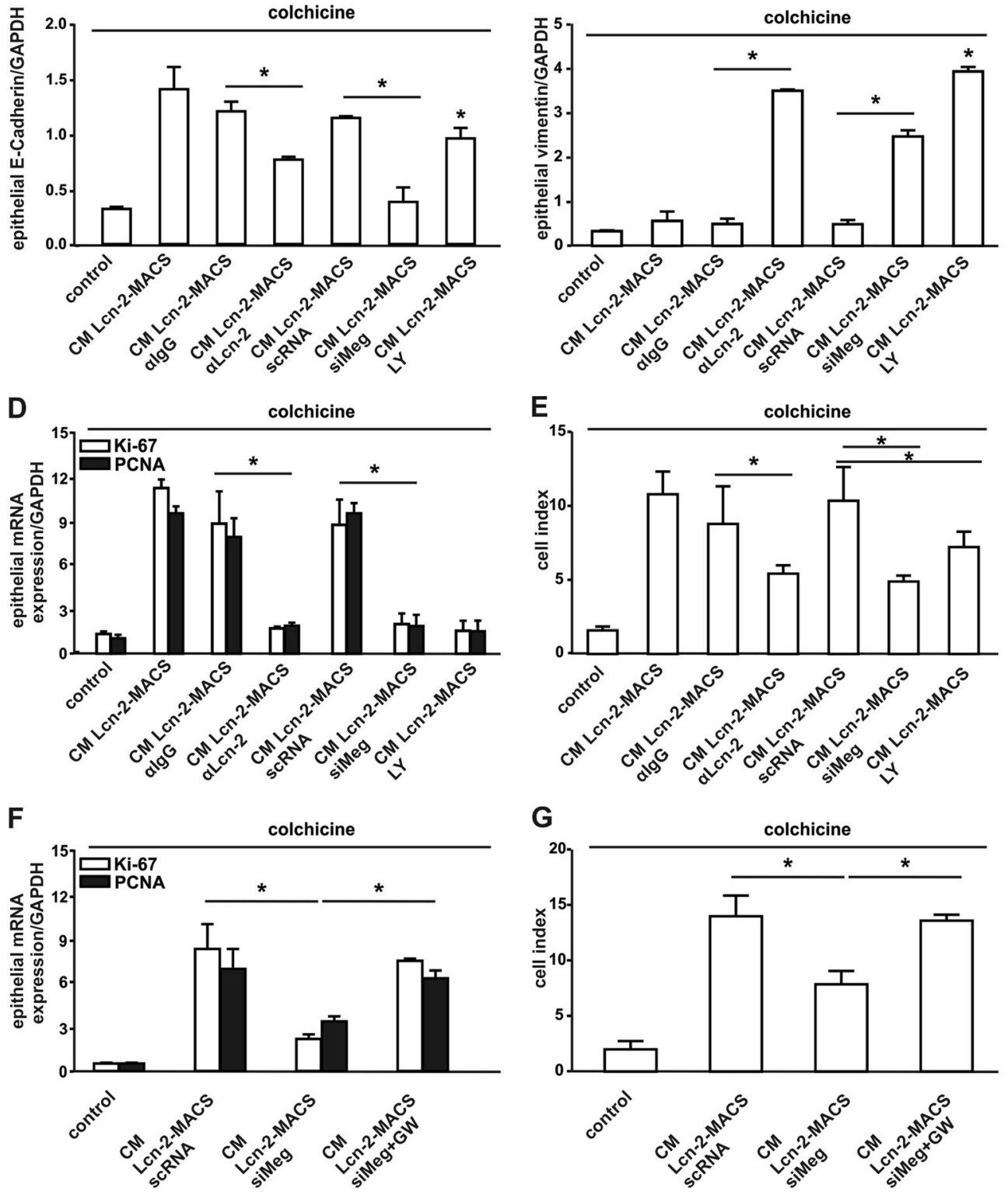

expression of Ki-67 and Pcna (Fig. 5d) and real-time proliferation measurements by applying xCELLigence (Fig. 5e). Apparently, the knockdown of megalin and inhibition of the
PI3K-Akt-pathway by LY294002 did not allow Len-2 to induce proliferation in renal epithelial cells. In order to analyse the role of megalin in defining Lcn-2-dependent 
Fig. 5 PI3K-Akt-pathway and megalin cooperate with Lcn-2 to induce proliferation and preserve epithelial phenotype. a Akt phosphorylation was detected by western blot analysis after stimulation with CM for 24 h. Total Akt was determined as control. The PI3K/Akt-pathway inhibitor LY294002 (10 $\mu \mathrm{M}$; Lcn-2-MACS + LY) was used as control. A representative picture of at least five independent experiments is given and quantification was performed using densitometrical measurements applying the Odyssey software. Data are means \pm S.E.M., $* p \leq 0.05$. b E-cadherin and c vimentin mRNA expression was quantified by qRT-PCR in NRK52e cells stimulated with CM of the previous groups as indicated. $n=6$, data are means \pm S.E.M., $* p \leq 0.05$. Proliferation measured by (d) mRNA expression of Ki-67 and Pcna as well as (e) xCELLigence in NRK52e cells stimulated with CM in the presence of either a knockdown of megalin or the inhibition of the Akt/ PI3K pathways using LY294002 $(10 \mu \mathrm{M}) . n=6$, data are means \pm S.E. M., ${ }^{*} p \leq 0.05$. f, $\mathbf{g}$ NRK-52e cells were stimulated with CM from Lcn2-overexpressing macrophages in the absence or presence of the PPAR $\gamma$ antagonist GW9662 (100 nM). Proliferation was measured by (f) mRNA analysis of $\mathrm{Ki}-67$ and Pcna as well as (g) using xCELLigence real-time measurements. $n=6$, data are means \pm S.E.M., ${ }^{*} p \leq$ 0.05

Ppary inhibition, we determined mRNA expression of the proliferation markers Pcna and Ki-67 (Fig. 5f) as well as real-time proliferation by the xCELLigence system (Fig. 5g). We observed that the presence of the PPAR $\gamma$ antagonist GW9662 (100 nM) induced the proliferative capacity in megalin-knockdown cells. A scheme summarizing the results of this study is given in Fig. 6 .

\section{Discussion}

In this study, we prove that Lcn-2 rescues epithelial cells from cell cycle arrest, promotes proliferation of damaged epithelial cells and preserves the cytoskeleton through the activation of PI3K-Akt dependent signalling and PPAR $\gamma$ inhibition.

Under the appropriate stimulus, macrophages may change their phenotype from a classically activated, proinflammatory to an alternative, anti-inflammatory one, thus supporting cell survival and proliferation, which finally leads to healing [20-23]. However, this is largely oversimplified and it is more reliable that different resident macrophage subsets of the tissue change in their phenotype and/or locally proliferate. Moreover, newly recruited monocytes that differentiate into macrophages upon entering the tissue enhance macrophage responses according to the stimulus encountered in their local microenvironment. Previous work of our group indicated that macrophages were able to promote repair under anti-inflammatory conditions. While it may be evident that these macrophages give rise to the expression of anti-inflammatory cytokines in order to promote epithelial recovery, they also foster the expression of pro-regenerative factors like Lcn-2. Results of the present study reinforce these findings and show that

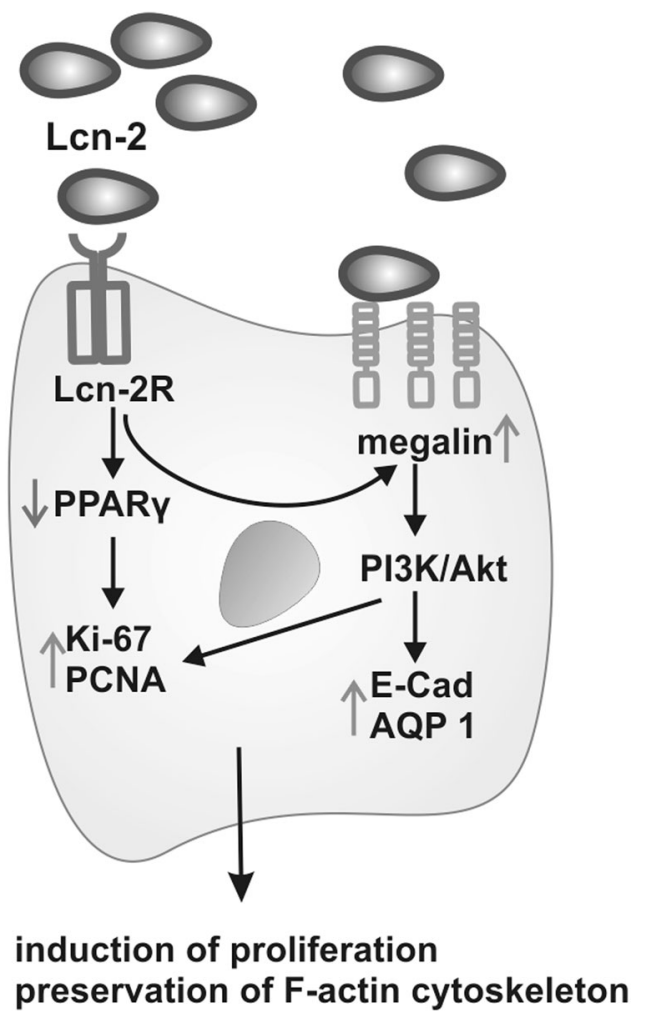

Fig. 6 Summarizing scheme macrophage-derived Lcn- 2 is taken up by its specific receptor Lcn-2R expressed on renal epithelial cells, leading to the inhibition of PPAR $\gamma$ and the subsequent promotion of cellular proliferation with enhanced expression of Ki-67 and PCNA. Lcn-2 also upregulates the receptor megalin, which serves as an unspecific receptor for Lcn-2. Downstream of megalin, the PI3K-Akt-pathway is activated and leads to further induction of epithelial proliferation

Lcn-2 is able to promote the re-entry of epithelial cells in active cell cycle and re-differentiation towards a healthy epithelial phenotype. This is in line with previous studies, reporting that Lcn-2 acts as a growth and differentiation factor in a variety of cell types [24].

In this study, we aimed at investigating the mechanistic insights of macrophage-derived Lcn-2 on renal epithelial cell proliferation. In an in vitro co-culture system employing bone marrow-derived macrophages, we showed that Lcn-2 induced cellular proliferation via activation of the PI3K-Akt signalling pathway that culminated in PPAR- $\gamma$ inhibition. Utilizing siRNA, we demonstrated a critical involvement of megalin in mediating downstream effects of Lcn-2 in renal epithelial cells.

Our results indicate that Lcn-2 also has a role in the preservation of F-actin associated to the cytoskeleton, which could explain its involvement in repair. In fact, we observed that Lcn-2 protein promotes the integrity of cell cytoskeleton by preserving homogenous distribution of the F-actin stress fibres. Previous studies on the role of macrophages in cell proliferation and repair have been centred 
on extracellular matrix remodelling $[25,26]$. Here, we focused on the ability of macrophages to rescue cells from cell cycle arrest and the subsequent reactivation of the cellular proliferative potential. Both processes are crucial to restore tissue integrity and homoeostasis[27]. One central question of the study was to identify how Len-2 stimulates pro-survival features in epithelial cells. A recent study suggested a role for Lcn-2 in PPAR $\gamma$ activation and function regarding insulin sensitization and fatty acid homoeostasis in high-fat diet-induced obesity [28]. In addition to its role in metabolism, PPAR $\gamma$ has been implicated in the control of the cell cycle and apoptosis. Treatment of tumour cells with PPAR $\gamma$ agonists led to cell cycle arrest. Furthermore, the activation of PPAR $\gamma$ promoted the induction of apoptosis $[29,30]$. Interestingly, we observed a significant inhibition of PPAR $\gamma$ activity upon Lcn-2 stimulation in epithelial cells. Therefore, it can be speculated that Lcn- 2 controls cell cycle reactivation through the inhibition of PPAR $\gamma$. Additionally, we found that Lcn-2 specifically acts on PPAR $\gamma$, whereas PPAR $\alpha$ or PPAR $\delta$ expression was not affected.

One decade ago, Devireddy et al. demonstrated that Lcn2 is internalized upon binding to its specific receptor Lcn-2R and is released to the cytoplasm by an endocytic delivery mechanism [31]. The existence of a specific receptor was first described in murine FL5.12 cells. The expression of the receptor conferred the ability to take up Lcn-2-bound iron and thus, to promote survival and cell growth. Now, we studied the expression of the specific Lcn-2 receptor in renal epithelial cells after co-culture with primary macrophages. We observed a significant upregulation of Lcn-2R expression in the presence of high levels of Lcn-2 released into the medium by macrophages, assuming that Lcn- 2 might stimulate the expression of its own receptor in renal epithelial cells. Furthermore, we hypothesize that Lcn-2 acts via binding to its specific receptor and triggers downstream pathways of proliferation and reactivation of the cell cycle. Apart from Lcn-2R, megalin is also described to serve as a receptor for Lcn-2. Our data confirm a significant upregulation of megalin under Lcn-2 incubation with downstream activation of the PI3K/Akt-pathway. Interestingly, megalin is a membrane glycoprotein, which not only serves as a receptor, but also adopts a pivotal role in determining functional responses in renal epithelial cells, thus representing a key feature of polarized renal epithelia. In this context, it may be hypothesized that Lcn-2 is a crucial as a pro-proliferative factor in order to rebuild damaged epithelia under pathological processes.

In conclusion, we found that Lcn-2 is a major player in rescuing epithelial cell cycle arrest and in the maintenance of the epithelial cell phenotype as well as the preservation of cytoskeleton structure. We identified one piece of the complex mechanism of macrophage-induced epithelial cell proliferation, involving Lcn-2-dependent inhibition of PPAR $\gamma$ downstream signalling, thereby defining the proliferation after damage in epithelial cells.

Acknowledgements The authors thank Angeles Muñoz for her excellent technical support. Financial support of this study came from grants from Fondo de Investigación Sanitaria (FIS) PI1701411 (awarded to A.S.) and SAF2015-67770-R from Ministerio de Economia y Competitividad (awarded to G.H.). A.S. was supported by Miguel Servet II contracting system (CPII 14/00026) and M.J. was supported by a grant from Fritz Thyssen Stiftung (Az.10.12.2.156) and a young researcher grant from Goethe-University Frankfurt (Focus Line B).

\section{Compliance with ethical standards}

Conflict of interest The authors declare that they have no conflict of interest.

\section{References}

1. Leoni G, Neumann P-A, Sumagin R, et al. Wound repair: role of immune-epithelial interactions. Mucosal Immunol. 2015;8: 959-68.

2. Duffield JS, Park KM, Hsiao L-L, et al. Restoration of tubular epithelial cells during repair of the postischemic kidney occurs independently of bone marrow-derived stem cells. J Clin Invest. 2005; 115:1743-55.

3. Lin SL, Li B, Rao S, et al. Macrophage Wnt7b is critical for kidney repair and regeneration. Proc Natl Acad Sci USA. 2010;107:4194-9.

4. Lee S, Huen S, Nishio H, et al. Distinct macrophage phenotypes contribute to kidney injury and repair. J Am Soc Nephrol. 2011;22:317-26.

5. Cakarova L, Marsh LM, Wilhelm J, et al. Macrophage tumour necrosis factor-a induces epithelial expression of granulocytemacrophage colony-stimulating factor impact on alveolar epithelial repair. Am J Respir Crit Care Med. 2009;180:521-32.

6. Mussar K, Tucker A, McLennan L, et al. Macrophage/epithelium cross-talk regulates cell cycle progression and migration in pancreatic progenitors. PLoS ONE. 2014;9:e89492.

7. Jung $M$, Weigert $A$, Tausendschön $M$, et al. Interleukin-10induced Neutrophil Gelatinase-Associated Lipocalin production in macrophages with consequences for tumor growth. Mol Cell Biol. 2012;32:3938-48.

8. Borregaard N, Cowland JB. Neutrophil gelatinase-associated lipocalin, a siderophore-binding eukaryotic protein. Biometals. 2006;19:211-5.

9. Jung M, Sola A, Hughes J, et al. Infusion of IL-10-expressing cells protects against renal ischemia through induction of lipocalin-2. Kidney Int. 2012;81:969-82.

10. Jung $M$, Brune $B$, Hotter $G$, et al. Lipocalin-2 contributes to ischemic resistance mechanisms by protecting from renal injury. Sci Rep. 2016;6:21950-61.

11. Wang G, Ma N, Meng L, et al. Activation of the phosphatidylinositol 3-kinase/Akt pathway is involved in lipocalin-2-promoted human pulmonary artery smooth muscle cell proliferation. Mol Cell Biochem. 2015;410:207-13.

12. Jin $\mathrm{D}$, Guo $\mathrm{H}, \mathrm{Bu} \mathrm{SY}$, et al. Lipocalin 2 is a selective modulator of and function in lipid homeostasis and energy expenditure. FASEB J. 2011;25:754-64.

13. Pereira VH, Marques F, Lages V, et al. Glucose intolerance after chronic stress is related with downregulated PPAR- $\gamma$ in adipose tissue. Cardiovasc Diabetol. 2016;15:114-9. 
14. Lu J, Shi J, Gui B, et al. Activation of PPAR- $\gamma$ inhibits PDGFinduced proliferation of mouse renal fibroblasts. Eur J Pharmacol. 2016;15:222-8

15. Sola A, Weigert A, Jung M, et al. Sphingosine-1-phosphate signalling induces the production of Lcn-2 by macrophages to promote kidney regeneration. J Pathol. 2011;225:597-608.

16. Kluth DC, Erwig LP, Pearce WP, et al. Gene transfer into inflamed glomeruli using macrophages transfected with adenovirus. Gene Ther. 2000;7:263-70.

17. Jung M, Oren B, Mora J, et al. Lipocalin 2 from macrophages stimulated by tumor cell-derived sphingosine 1-phosphate promotes lymphangiogenesis and tumor metastasis. Sci Signal. 2016;9:ra64.

18. Jung M, Hotter G, Vinas JL, et al. Cisplatin upregulates mitochondrial nitric oxide synthase and peroxynitrite formation to promote renal injury. Toxicol Appl Pharmacol. 2009;234:236-46.

19. Zhang J, Wu Y, Zhang Y, et al. The role of lipocalin 2 in the regulation of inflammation in adipocytes and macrophages. Mol Endocrinol. 2008;22:1416-26.

20. Arnold L, Henry A, Poron F, et al. Inflammatory monocytes recruited after skeletal muscle injury switch into antiinflammatory macrophages to support myogenesis. J Exp Med. 2007;204: 1057-69.

21. Ricardo SD, van Goor H, Eddy AA. Macrophage diversity in renal injury and repair. J Clin Invest. 2008;118:3522-30.

22. Lucas T, Waisman A, Ranjan R, et al. Differential roles of macrophages in diverse phases of skin repair. J Immunol. 2010;184:3964-77.

23. Sica A, Erreni M, Allavena P, et al. Macrophage polarization in pathology. Cell Mol Life Sci. 2015;72:4111-26.
24. Schmidt-Ott KM, Mori K, Li JY, et al. Dual action of neutrophil gelatinase-associated lipocalin. J Am Soc Nephrol. 2007;18: 407-13.

25. Wilson HM, Barker RN, Erwig LP. Macrophages: promising targets for the treatment of atherosclerosis. Curr Vasc Pharmacol. 2009;7:234-43.

26. Madsen DH, Leonard D, Masedunskas A, et al. M2-like macrophages are responsible for collagen degradation through a mannose receptor-mediated pathway. J Cell Biol. 2013; 202:951-66.

27. Yang L, Besschetnova TY, Brooks CR, et al. Epithelial cell cycle arrest in G2/M mediates kidney fibrosis after injury. Nat Med. 2010;16:535-44.

28. Jin $\mathrm{D}$, Guo H, Bu S, et al. Lipocalin 2 is a selective modulator of peroxisome proliferator-activated receptor-gamma activation and function in lipid homeostasis and energy expenditure. FASEB J. 2011;25:754-64.

29. Elstner E, Muller C, Koshizuka K, et al. Ligands for peroxisome proliferator-activated receptorgamma and retinoic acid receptor inhibit growth and induce apoptosis of human breast cancer cells in vitro and in BNX mice. Proc Natl Acad Sci USA. 1998;95:8806-11.

30. Padilla J, Kaur K, Cao HJ, et al. Peroxisome proliferator activator receptor-gamma agonists and 15-deoxy-Delta $(12,14)(12,14)-P G J$ (2) induce apoptosis in normal and malignant B-lineage cells. J Immunol. 2000;165:6941-8.

31. Devireddy LR, Gazin C, Zhu X, et al. A cell-surface receptor for lipocalin $24 \mathrm{p} 3$ selectively mediates apoptosis and iron uptake. Cell. 2005;123:1293-305. 